\title{
Direct Incorporation of Scattering Parameters Into the FDTD Algorithm
}

\author{
Zhiguo Su, Student Member, IEEE, and Thomas J. Brazil, Fellow, IEEE
}

\begin{abstract}
A direct method for incorporating S-parameters into an finite-difference time-domain (FDTD) algorithm is presented in this paper. The frequency-domain S-parameters of an arbitrary network to be embedded within an electromagnetic system are represented as a series of modified impulse responses using a nonuniform discrete-time technique. In this way, the currents passing through the embedded network can be calculated using a convolution in the time-domain. The calculated currents are used to update the electric fields at the locations occupied by the embedded network according to Ampere's law. The simulation results using this method have been compared with the results simulated by Lumped-Element FDTD method (LE-FDTD), showing very good agreement. The method presented here is highly accurate and stable, and is suitable for use in quite general cases, especially where the embedded network cannot easily be represented by lumped elements.
\end{abstract}

Index Terms-Finite-difference time-domain (FDTD), incorporating, nonuniform, S-parameters.

\section{INTRODUCTION}

A S THE SIGNAL frequencies used in modern microwave electronics have increased rapidly, the finite-difference time-domain (FDTD) method [1], [2] has been widely used to analyze many different types of electromagnetic problems. Research has been reported that extends the basic FDTD method to hybrid electromagnetic systems involving active and passive elements [3]-[7], where circuit elements such as resistors, capacitors, inductors, diodes, and transistors are treated as sub-grid models on the FDTD meshes. The most popular method of embedding elements into FDTD meshes is so-called lumped-element FDTD method (LE-FDTD), which embeds lumped circuit elements into FDTD in terms of Ampere's current law. Indeed, simulation results obtained using the LE-FDTD method show very good agreement with practical measurements.

However in many cases, the embedded elements are linear time-invariant networks obtained from separate simulations or measurements, and these embedded networks cannot easily be represented in terms of a lumped element equivalent circuit model. It would be preferable, therefore, if a high-level description of such networks, typically based on scattering parameters (S-parameters), could be easily and directly interfaced with the FDTD algorithm. Simplistically, this can be seen as requiring

Manuscript received July 15, 2008; revised October 09, 2008. Current version published April 08, 2009. This work was supported by Science Foundation Ireland.

The authors are with the School of Electrical, Electronic and Mechanic Engineering, University College Dublin, Dublin, Ireland (e-mail: zhiguo.su@ucd.ie; tom.brazil@ucd.ie).

Digital Object Identifier 10.1109/TAP.2009.2015830 an inversion of the S-parameters to the time-domain using some form of numerical implementation of the Fourier Transform. In practice, this is difficult to achieve for data known only up to some maximum frequency $f_{m}$ that is not naturally periodic.

A new method is presented in this work enabling the direct incorporation of a network described by S-parameters into the FDTD algorithm. Firstly, in Section II it is shown how the frequency-domain S-parameters of an arbitrary network can be represented efficiently and accurately as modified impulse responses in the time-domain using a nonuniform discrete-time technique [8], [9]. This method avoids the serious inaccuracies that would otherwise occur when wideband nonperiodic S-parameter functions are transferred into the time-domain by the inverse fast fourier transform (IFFT) algorithm. Section III shows how these modified impulse responses may be incorporated into the FDTD algorithm using convolution to modify the updating equations of the FDTD method according to Ampere's current law. Finally, in Section IV, the results simulated by this method have been compared with independent results obtained by the LE-FDTD method, showing very good mutual agreement. The conclusions are given in Section V.

\section{Non-Uniform Discrete-Time TeChNique}

Because FDTD is fundamentally a time-domain algorithm, the problem addressed by this paper requires that frequencydomain S-parameters must be converted into the time-domain. However the S-parameters are usually available as nonperiodic data specified only up to some maximum frequency $f_{m}$ : it is a nontrivial task to achieve the required conversion with a high degree of accuracy and efficiency. As already noted, strategies such as using a "window" function to suppress the given function outside the known band can create serious inaccuracies. Periodic extension appears attractive but seems very difficult to manage in the general case without introducing serious discontinuities in either the magnitude or phase at the boundaries of the periodic interval.

In order to overcome this problem, a nonuniform discretetime technique can be used to obtain representation of the S-parameters in the time-domain [8], [9]. As an example, consider a given one-port S-parameter $\mathrm{S}_{11}$, which is expressed as a Hermitian function by (1), where $S_{11}^{r}(f)$ and $S_{11}^{i}(f)$ are the real part and imaginary part of $S_{11}(f)$, respectively, and $\mathrm{f}_{\mathrm{m}}$ is the maximum (positive) frequency

$$
S_{11}(f)= \begin{cases}S_{11}^{r}(f)+j \cdot S_{11}^{i}(f) & \left(0 \leq f \leq f_{m}\right) \\ S_{11}^{r}(f)-j \cdot S_{11}^{i}(f) & \left(-f_{m} \leq f<0\right) .\end{cases}
$$

A direct periodic extension of the S-parameters beyond the known range $\left[-f_{m}, f_{m}\right]$ will generally introduce major complex- 


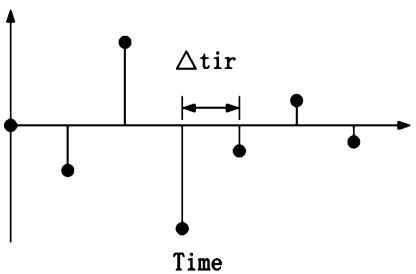

Fig. 1. Time-domain representation of $F_{11}(f)$.

valued discontinuities at each boundary frequency. To avoid this kind difficulty we introduce the following equation:

$$
F_{11}(f)=\left[S_{11}(f)-K\right] \cdot e^{-j 2 \pi f \tau}
$$

where $K$ and $\tau$ are real-valued unknowns which are chosen to ensure that $F_{11}(f)$ is both period and continuous at the maximum frequency as well as possessing a zero-valued impulse response representation at zero time when converted to the (discrete) time-domain. Hence, the following two conditions must be satisfied simultaneously.

1) $\operatorname{Im}\left[F_{11}\left(f_{m}\right)\right]=0$. For a Hermitian function this is sufficient to avoid a discontinuity in $F_{11}(f)$ at $\mathrm{f}=\mathrm{f}_{\mathrm{m}} \cdot F_{11}(f)$ is thus continuous and periodic in a complex-valued sense and may be represented efficiently by a discrete-time sequence of impulse response weights separated by $\Delta$ tir $=$ $1 /\left(2 f_{m}\right)$.

2) The impulse response calculated as a result of condition (1) at time $=0$ is forced to be exactly zero.

Taking these conditions into account, two equations can be derived to determine values of $K$ and $\tau \in\left[0,1 /\left(2 f_{m}\right)\right]$ such that

$$
\begin{aligned}
& \sum_{n=1}^{N-1} 2 \cdot\left\{\begin{array}{c}
\left(S_{11}^{r}(n \Delta f)-K\right) \cdot \cos (2 \pi n \Delta f \tau) \\
+S_{11}^{i}(n \Delta f) \cdot \sin (2 \pi n \Delta f \tau)
\end{array}\right\} \\
& \quad+S_{11}^{r}(0)-K \\
& \quad+\left(S_{11}^{r}\left(f_{m}\right)-K\right) \cdot \cos \left(2 \pi f_{m} \tau\right) \\
& \quad+S_{11}^{i}\left(f_{m}\right) \cdot \sin \left(2 \pi f_{m} \tau\right)=0 \\
& \quad \text { and } \\
& K=S_{11}^{r}\left(f_{m}\right)-S_{11}^{i}\left(f_{m}\right) / \tan \left(2 \pi f_{m} \tau\right)
\end{aligned}
$$

where $\Delta f$ is the interval of samples in frequency-domain, and the S-parameters are specified at $\mathrm{N}+1$ equally-spaced samples within the range $\left[0, f_{m}\right]$.

The resulting time-domain representation of $F_{11}(f)$ may resemble that shown in Fig. 1. However, our initial objective was to obtain a discrete-time representation of the S-parameters. This is achieved as follows.

1) Remove the additional phase shift introduced by the exponential term in (2). This corresponds to shift the entire response in Fig. 1 by an amount $\tau$ to the left, i.e., in the direction of negative time, and explains the need for condition (2), since moving the first, zero-valued sample into negative time region does not then led to a violation of causality.

2) If $\mathrm{K}$ is nonzero, an additional impulse at time zero should be added into the representation of $S_{11}(f)$ and the value of impulse is $\mathrm{K}$. The resulting discrete-time representation

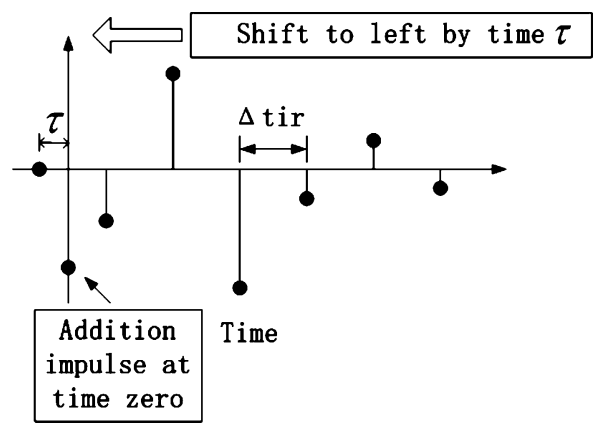

Fig. 2. Time-domain representation of S-parameter $\mathrm{S}_{11}(\mathrm{f})$ derived from response Fig. 1.

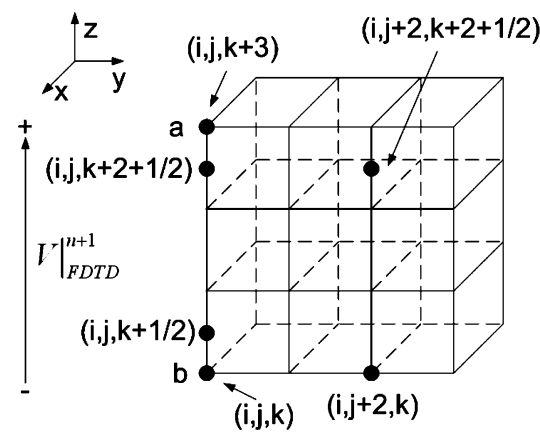

Fig. 3. Network spanning several cells oriented in the $\mathrm{z}$ direction, where $i, j$, and $k$ are the coordinates indicating the vertex of a space cell.

of $S_{11}(f)$ is as shown in Fig. 2. The first interval is shorter than other uniform interval. Therefore, the representation of $S_{11}(f)$ is nonuniform discrete-time impulse response and is described here as the modified impulse response.

Solving (3) is a straightforward numerical exercise in finding a bracketed root of an algebraic nonlinearity. The modified impulse response will be used to incorporate the S-parameters into FDTD algorithm in the next section.

\section{INCORPORATING TIME-DOMAIN REPRESENTATION OF NETWORK S-PARAMETERS INTO THE FDTD ALGORITHM}

The link between the electromagnetic fields in the FDTD cells and the current through the embedded network is provided by Ampere's law. The method of embedding circuit currents into the FDTD algorithm at time $(n+1)$ was proposed by Li [10], and appears to be very stable. The integral form of Ampere's law is applied to obtain the FDTD updating equations at the location at which the network is embedded

$$
\begin{aligned}
\left.\oint_{C} H\right|^{n+1 / 2} \cdot d l=\int_{S} \varepsilon & \frac{\left.\partial E\right|^{n+1 / 2}}{\partial t} d s \\
& +\left.\int_{S} \sigma E\right|^{n+1 / 2} d s+\left.\int_{S} J\right|_{\text {net }} ^{n+1} d s
\end{aligned}
$$

where $\oint_{C}$ and $\int_{S}$ are the contour integration along the path which encloses the current of the embedded network and the surface integration of the area where the current of the network passes through, respectively. It is assumed that the network occupies several FDTD cells and is oriented along the $\mathrm{z}$ direction as shown in Fig. 3. 
Discretizing (5) gives

$$
\begin{aligned}
& \left(\left.H_{y}\right|_{i+1 / 2, j, k+1 / 2} ^{n+1 / 2}-\left.H_{y}\right|_{i-1 / 2, j, k+1 / 2} ^{n+1 / 2}\right) \Delta y \\
& \text { - }\left(\left.H_{x}\right|_{i, j+1 / 2, k+1 / 2} ^{n+1 / 2}-\left.H_{x}\right|_{i, j-1 / 2, k+1 / 2} ^{n+1 / 2}\right) \Delta x \\
& =\left[\varepsilon \frac{\left.E_{z}\right|_{i, j, k+1 / 2} ^{n+1}-\left.E_{z}\right|_{i, j, k+1 / 2} ^{n}}{\Delta t}\right. \\
& \left.+\sigma \frac{\left.E_{z}\right|_{i, j, k+1 / 2} ^{n+1}+\left.E_{z}\right|_{i, j, k+1 / 2} ^{n}}{2}\right] \Delta x \Delta y+\left.I\right|_{\text {net }} ^{n+1}
\end{aligned}
$$

where the superscripts and subscripts in (6) stand for the time and spatial coordinates of the electromagnetic fields respectively. It should be noted that the electric fields at time $(n+1 / 2)$ are calculated using the values at time (n) and $(\mathrm{n}+1)$.

Assuming that the permittivity and the conductivity of the medium are real and frequency-independent values, equation (6) can be simplified as following:

$$
\left.E_{z}\right|_{i, j, k+1 / 2} ^{n+1}=\left.K\right|_{i, j, k+1 / 2} ^{n}-\left.\left.I\right|_{\text {net }} ^{n+1} \Gamma\right|_{i, j, k}
$$

where

$$
\begin{aligned}
& \left.\Gamma\right|_{i, j, k} \\
& =\frac{1}{\left(\frac{\varepsilon_{i, j, k}}{\Delta t}+\frac{\sigma_{i, j, k}}{2}\right) \Delta x \Delta y} \\
& \left.T\right|_{i, j, k+1 / 2} ^{n+1 / 2} \\
& =\left(\begin{array}{ll}
\left.H_{y}\right|_{i+1 / 2, j, k+1 / 2} ^{n+1 / 2}-\left.H_{y}\right|_{i-1 / 2, j, k+1 / 2} ^{n+1 / 2}
\end{array}\right) \Delta y \\
& \text { - }\left(\left.H_{x}\right|_{i, j+1 / 2, k+1 / 2} ^{n+1 / 2}-\left.H_{x}\right|_{i, j-1 / 2, k+1 / 2} ^{n+1 / 2}\right) \Delta x \\
& \left.K\right|_{i, j, k+1 / 2} ^{n} \\
& =\left.\Gamma\right|_{i, j, k}\left[\left.T\right|_{i, j, k+1 / 2} ^{n+1 / 2}\right. \\
& \left.+\left.\left(\frac{\varepsilon_{i, j, k}}{\Delta t}-\frac{\sigma_{i, j, k}}{2}\right) \Delta x \Delta y E_{z}\right|_{i, j, k+1 / 2} ^{n}\right] .
\end{aligned}
$$

A closer look at $\left.\Gamma\right|_{i, j, k}$ reveals that it can be interpreted as the cell's sheet resistivity along the $x-y$ plane at point $i, j, k$. Also, $\left.K\right|_{i, j, k+1 / 2} ^{n}$ is the voltage that results due to the circulating magnetic field and the potential difference in the electric field intensity between the two faces of the cell.

The voltage $\left.V\right|_{\mathrm{FDTD}} ^{n+1}$ from a to $\mathrm{b}$ in Fig. 3 is computed by the integration of the Ez components along the path from $a$ to $b$. For example, only considering the cells of the left column, the equation which relates the voltage to $\mathrm{Ez}$ is as follows:

$$
\begin{aligned}
\left.V\right|_{\mathrm{FDTD}} ^{n+1} & =\left.\sum_{m=k}^{k+2} E_{z}\right|_{i, j, m+1 / 2} ^{n+1} \Delta z \\
& =\left.\sum_{m=k}^{k+2} K\right|_{i, j, m+1 / 2} ^{n} \Delta z-\left.\left.I\right|_{\mathrm{net}} ^{n+1} \sum_{m=k}^{k+2} \Gamma\right|_{i, j, m} \Delta z .
\end{aligned}
$$

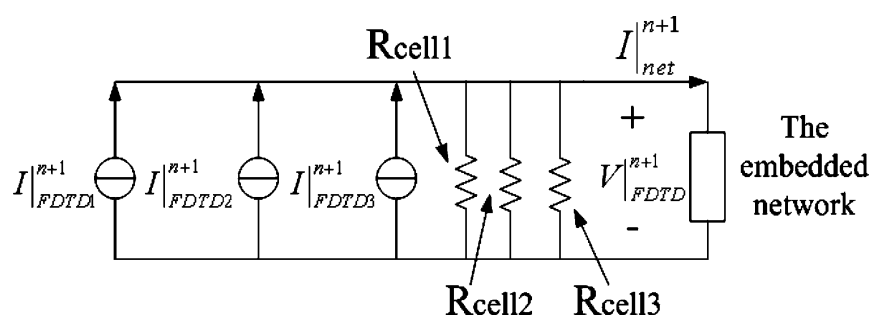

Fig. 4. Norton equivalent circuit derived from Fig. 3 for the network embedded within the FDTD solution.

A Norton equivalent circuit can be derived from (8):

$$
\left.I\right|_{\mathrm{FDTD} 1} ^{n+1}=\frac{\left.V\right|_{\mathrm{FDTD}} ^{n+1}}{R_{\mathrm{cell} 1}}+\left.I\right|_{\mathrm{net}} ^{n+1}
$$

where

$$
\begin{aligned}
R_{\text {cell1 }} & =\left.\sum_{m=k}^{k+2} \Gamma\right|_{i, j, m} \Delta z, \\
\left.I\right|_{\mathrm{FDTD} 1} ^{n+1} & =\left.\sum_{m=k}^{k+2} K\right|_{i, j, m+1 / 2} ^{n} \Delta z / R_{\text {cell1 }} .
\end{aligned}
$$

In Fig. 3 there are three columns FDTD cells, so the whole Norton equivalent circuit is described in Fig. 4.

As shown in Fig. 4, the three current sources are time-variant signals, and the three internal resistors are time-invariant because the permittivity and the conductivity of the medium are assumed to be frequency-independent. According to the definition of S-parameters, the frequency-domain equation corresponding to Fig. 4 can be derived as following:

$\frac{R_{\text {cell_SUM }}\left[I_{\text {FDTD_SUM }}(f)-I_{\text {net }}(f)\right]-Z_{0} I_{\text {net }}(f)}{R_{\text {cell_SUM }}\left[I_{\text {FDTD_SUM }}(f)-I_{\text {net }}(f)\right]+Z_{0} I_{\text {net }}(f)}=S(f)$

where $I_{\text {FDTD_SUM }}, R_{\text {cell_SUM }}$ are the sums of the current sources and the resistances in parallel, respectively. $Z_{0}$ is the reference impedance whose typical value of $50 \mathrm{Ohms}$.

The previous Section showed how the time-domain representation of the S-parameters of the embedded network, could be found as a series of samples in the form of a modified impulse response, denoted here by $\mathrm{s}(\mathrm{t})$. Therefore, the frequency-domain expression (10) can be converted to (11) in the time-domain

$$
\begin{aligned}
& R_{\text {cell_SUM }} I_{\text {FDTD_SUM }}(t) \\
& -R_{\text {cell_SUM }} I_{\text {net }}(t)-Z_{0} I_{\text {net }}(t) \\
& \quad=\left[R_{\text {cell_SUM }} I_{\text {FDTD_SUM }}(t)\right. \\
& \left.-\left(R_{\text {cell_SUM }}-Z_{0}\right) I_{\text {net }}(t)\right] * s(t)
\end{aligned}
$$

where the operator * denotes convolution, and to simplify notation $I_{\text {net }}(n)$ presents $\left.I\right|_{\text {net }} ^{n}$ at time $\mathrm{n}$. Therefore, the current 


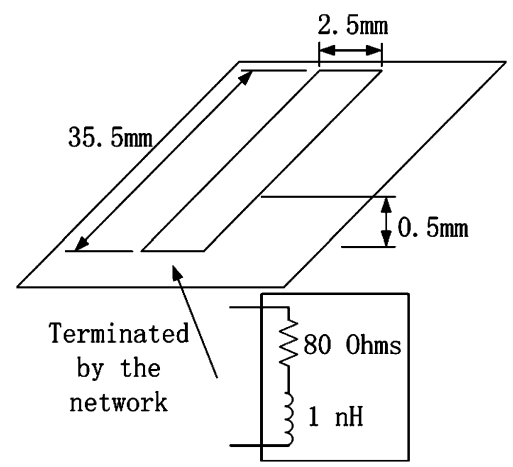

Fig. 5. Microstrip line terminated by a network which is composed of a resistor and an inductor in series.

passing through the network at time $\mathrm{n}+1,\left.I\right|_{\text {net }} ^{n+1}$, can be derived from the previous $\mathrm{I}_{\text {FDTD_SUM }}$, and $\left.I\right|_{\text {net }} ^{n}$.

$$
\begin{aligned}
\left.I\right|_{\text {net }} ^{n+1}= & I_{\text {net }}(n+1) \\
= & \frac{R_{\text {cell_SUM }}[s(0)-1] I_{\text {FDTD_SUM }}(n+1)}{\left[R_{\text {cell_SUM }}-Z_{0}\right] s(0)-\left(R_{\text {cell_SUM }}+Z_{0}\right)} \\
& \quad+\frac{\sum_{q=1}^{n+1} s(q) v(n+1-q)}{\left[R_{\text {cell_SUM }}-Z_{0}\right] s(0)-\left(R_{\text {cell_SUM }}+Z_{0}\right)}
\end{aligned}
$$

where

$$
\begin{aligned}
v(n+1-q)=R_{\text {cell_SUM }} & I_{\text {FDTD_SUM }}(n+1-q) \\
& -\left(R_{\text {cell_SUM }}-Z_{0}\right) I_{\text {net }}(n+1-q)
\end{aligned}
$$

It seems from (12) that the convolution has to be carried out over all time, but the embedded wideband network is here represented by a discrete-time function of comparatively short duration. Therefore, the number of multiplications actually necessary for the convolution in (12) is much less than $\mathrm{n}$, as are the dimensions of the needed historic data records of $I_{\text {net }}(t)$ and $I_{\text {FDTD_SuM }}(t)$. Assuming the number of samples from the first to the last nonzero sample of the modified impulse response is $L,(12)$ can be replaced by the simpler (13)

$$
\begin{aligned}
& \left.I\right|_{\text {net }} ^{n+1}=I_{\text {net }}(n+1) \\
& =\frac{R_{\text {cell_SUM }}[s(0)-1] I_{\text {FDTD_SUM }}(n+1)}{\left[R_{\text {cell_SUM }}-Z_{0}\right] s(0)-\left(R_{\text {cell_SUM }}+Z_{0}\right)} \\
& \quad+\frac{\sum_{q=1}^{L-1} s(q) v(n+1-q)}{\left[R_{\text {cell_SUM }}-Z_{0}\right] s(0)-\left(R_{\text {cell_SUM }}+Z_{0}\right)}
\end{aligned}
$$

Finally the calculated result $\left.I\right|_{\text {net }} ^{n+1}$ is inserted back into (6) to update the electric fields at the location where the network is embedded, and the normal FDTD updating equations are used to update the electric fields everywhere else.

\section{VERIFICATION}

To verify the method proposed in this study, a simple microstrip line ended by a network which is a resistor connecting an inductor in series, is presented as shown in Fig. 5. To simplify
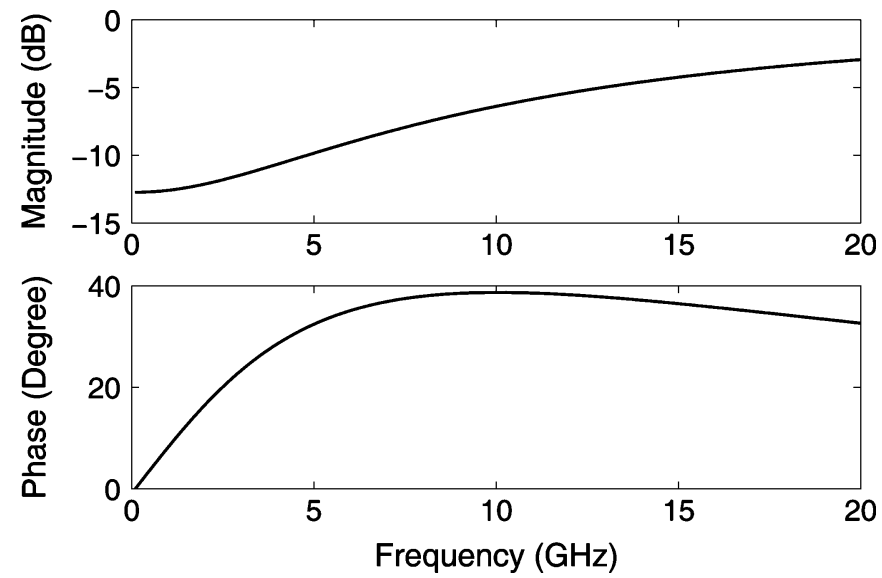

Fig. 6. The S-parameters of the embedded network.

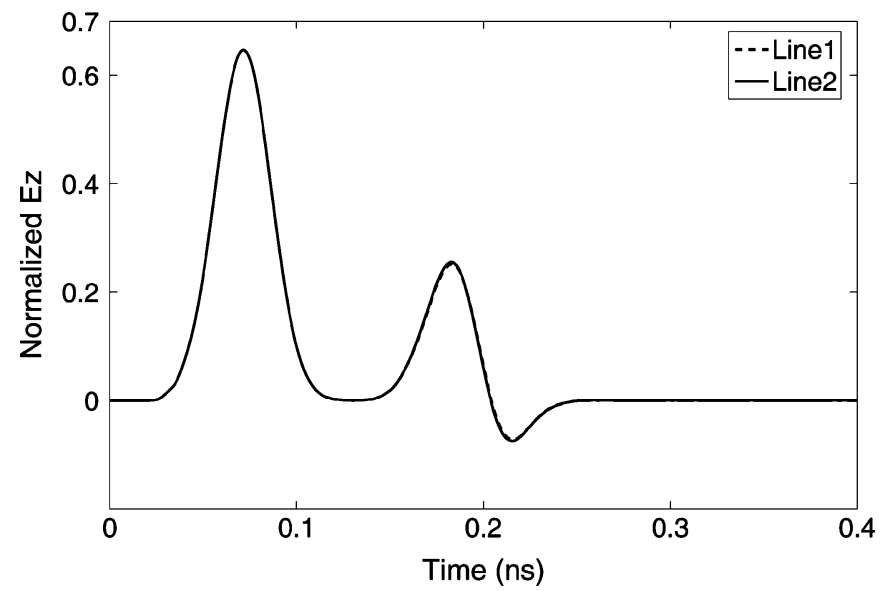

Fig. 7. The simulation results (Line1 is the simulation result by LE-FDTD method, and Line 2 is the simulation by the method in this study. The normalized Ez equals $\mathrm{Ez} \sqrt{\varepsilon_{0} / \mu_{0}}$, where $\varepsilon_{0}$ and $\mu_{0}$ are the permittivity and the permeability in vacuum respectively). The two results show very good agreement, and from the above figure can be seen virtually to coincide.

the simulation, the medium beneath and surround the microstrip line is air, and the distance between the ground and the strip is $0.5 \mathrm{~mm}$. The width of the strip is $2.5 \mathrm{~mm}$. The network terminating the microstrip line is a resistor of $80 \mathrm{Ohms}$ connecting a inductor of $1 \mathrm{nH}$ in series, and the reflection S-parameter of this network is shown in Fig. 6, where it is clear that the phase at the maximum frequency, $20 \mathrm{GHz}$, is nonzero so that nonuniform discrete-time technique must be use to convert the S-parameters into the time-domain to obtain the modified impulse response.

The FDTD cell size within the simulation is $0.5 \mathrm{~mm}$ $\times 0.5 \mathrm{~mm} \times 0.5 \mathrm{~mm}$. Twelve perfectly matched layers (PML) are placed at the boundary of the computational domain to mimic the open-region problem. The embedded network spans the same width as the width of the microstrip line, so that the cells occupied the network are 5 cells (x direction) $\times 1$ cell $(y$ direction $) \times 1$ cell ( $\mathrm{z}$ direction) as shown in Fig. 5 .

The source of the simulation is a Gaussian pulse which is $22.5 \mathrm{~mm}, 45$ cells, far away from the end of the strip. Two simulations were run with LE-FDTD method and the method used in this study. The normalized Ez of the center cell is calculated, which is located just beneath the strip and a distance of $15 \mathrm{~mm}$, or 30 cells, from the end of strip. A comparison of the results is given in Fig. 7, showing very close agreement. 


\section{CONCLUSION}

The method presented in this study can incorporate generalized S-parameters into the FDTD algorithm directly. Firstly the frequency-domain S-parameters of the embedded network are represented as a modified impulse response very accurately by the nonuniform discrete-time technique. Secondly the current passing through the network at time $\mathrm{n}+1$ is calculated by convolution processing. Finally the calculated current is used to update the electric fields by the Ampere's law.

The simulation result by this method has been compared with the result simulated by LE-FDTD method. The comparison verifies that the method in this study is highly accurate. Furthermore, the method proposed in this study can be used for very general cases, especially for networks which can not easily or efficiently be represented by combinations of lumped elements. In this study an example of a one-port S-parameter network embedded into the FDTD algorithm is shown to illustrate the analysis. Nevertheless this method can easily be generalized to the case of multiple-port S-parameter networks interfaced with the FDTD algorithm.

\section{REFERENCES}

[1] K. S. Yee, "Numerical solution of initial boundary value problems involving Maxwell's equations in isotropic media," IEEE Trans. Antennas Propag., vol. AP-14, pp. 302-307, May 1966.

[2] A. Taflove, Computational Electrodynamics: The Finite-Difference Time-Domain Method, 3rd ed. Norwood, MA: Artech House, 2005.

[3] W. Sui, D. A. Christensen, and C. H. Durney, "Extending the two-dimensional FD-TD method to hybrid electromagnetic systems with active and passive lumped elements," IEEE Trans. Microw. Theory Tech., vol. 40, no. 4, pp. 724-730, Apr. 1992.

[4] B. Toland, J. Lin, B. Houshmand, and T. Itoh, "FDTD analysis of an active antenna," IEEE Microw. Guided Wave Lett., vol. 3, no. 11, pp. 423-425, Nov. 1993.

[5] C. N. Kuo, S. T. Chew, B. Houshmand, and T. Itoh, "FDTD simulation of a microwave amplifier," in IEEE MTT-S Int. Microw. Symp. Dig., 1995, pp. 357-360.

[6] V. A. Thomas, M. E. Jones, M. J. Piket-May, A. Taflove, and E. Harrigan, "The use of SPICE lumped circuits as sub-grid models for FD-TD analysis," IEEE Microw. Guided Wave Lett., vol. 4, pp. 141-143, May 1994.
[7] M. Piket-May, A. Taflove, and J. Baron, "FDTD modeling of digital signal propagation in 3-D circuits with passive and active loads," IEEE Trans. Microw. Theory Tech., vol. 42, no. 8, pp. 1514-1523, Aug. 1994.

[8] T. J. Brazil, "Accurate and efficient incorporation of frequency-domain data within linear and nonlinear time-domain transient simulation," in Proc. IEEE Microw. Theory MTT-S Int. Microw. Symp. Dig., Jun. 2005, pp. 4-.

[9] Z. Su and T. J. Brazil, "Transient model using cascaded ideal transmission lines for UWB antenna for Co-simulation with circuits," in Proc. IEEE Microw. Theory MTT.-S Int. Microw. Symp. Dig., Jun. 2007, pp. 2035-2038.

[10] T. Li, "FDTD-based full wave co-simulation model for hybrid electromagnetic systems," Ph.D. dissertation, Dept. Elect. Comp. Eng., Inst. Technol., NJ, May 1999.

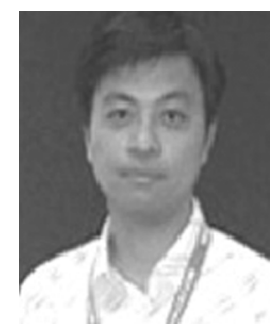

Zhiguo Su (M'07) was born in China, in 1977. He received the B.E.E. degree and the M.E.E. from the Southeast University, Nanjing, China, in 1999 and 2005 , respectively. He is currently working toward the Ph.D. degree in the School of Electrical, Electronic and Mechanic, University College Dublin (UCD), Dublin, Ireland.

$\mathrm{He}$ is currently with the RF and Microwave Research Group, UCD. His research interests include microstrip and UWB antennas, convolution-based $\mathrm{CAD}$ simulation techniques, signal integrity, full wave analyses of hybrid electromagnetic structures and dispersive media.

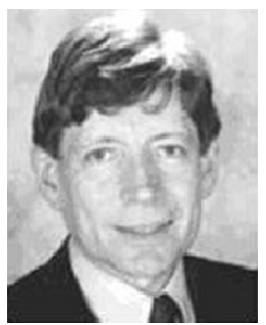

Thomas J. Brazil (M'86-SM'02-F'04) received the B.E. degree in electrical engineering from University College Dublin (UCD), Dublin, Ireland, in 1973, and the Ph.D. degree in electronic engineering from the National University of Ireland, Dublin, in 1977.

He then joined Plessey Research, Caswell, U.K., where he was involved with microwave subsystem development prior to returning to UCD in 1980. He is currently a Professor of electronic engineering and holds the Chair of Electronic Engineering at UCD. His research interests are in the fields of nonlinear modeling and characterization techniques at the device, circuit, and system levels. He also has interests in nonlinear simulation algorithms and several areas of microwave subsystem design and applications. He has authored or coauthored numerous publications in the international scientific literature in these fields.

Prof. Brazil is a Fellow of Engineer Ireland and a Member of the Royal Irish Academy. From 1998 to 2001, he was an IEEE Microwave Theory and Techniques Society (IEEE MTT-S) Worldwide Distinguished Lecturer in high-frequency computer-aided design (CAD) applied to wireless systems. He is currently a member of the IEEE MTT-1 Technical Committee on CAD. 